\title{
Phase-I Trigger Readout Electronics Upgrade of the ATLAS Liquid-Argon Calorimeters
}

Tatsuya Mori (Univ. of Tokyo, JP),

on behalf of the ATLAS Liquid Argon Calorimeter Group 


\section{Table of Contents}

- Overview : Phase-I upgrade of the ATLAS LAr Calorimeter readout

- Overview : The role of custom developed hardware for readout

- Performance results of prototype boards in the Demonstrator System 
ATLAS LAr Calorimeter

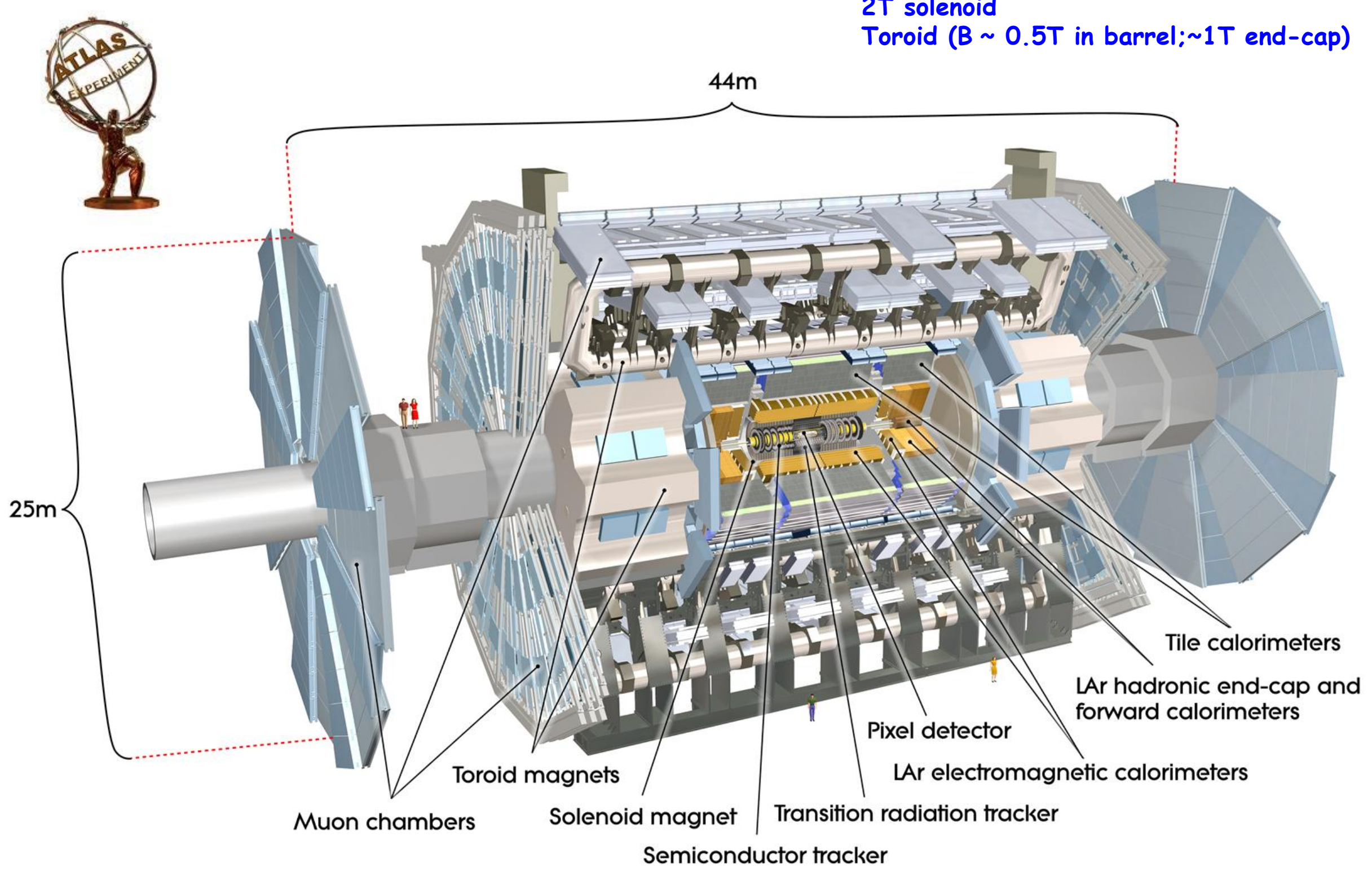

7000 tons

88 Million channels $3000 \mathrm{~km}$ of cables $2 \mathrm{~T}$ solenoid

Toroid ( 0.5T in barrel; 1T end-cap) 


\section{Phase I Upgrade of LAr Calorimeter Readout}

\section{Purpose :}

providing higher-granularity, higher-resolution, and longitudinal shower information from the calorimeter to the Level-1 trigger processors

\section{Strategy :}

increasing granularity 10 times by changing from Trigger Tower to Super Cell readout (will be done during 2018)

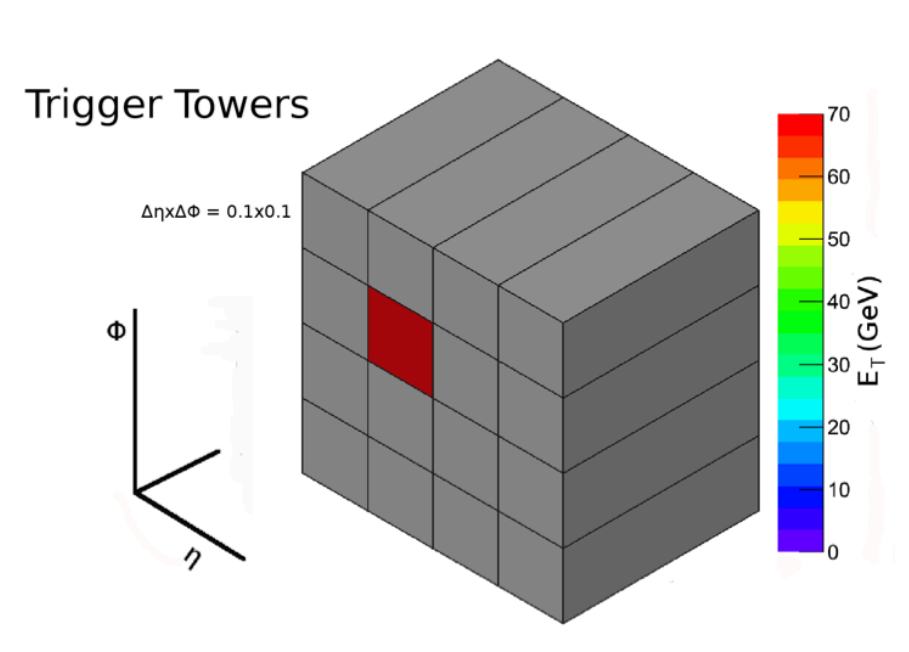

Trigger Tower :

sums the energy deposition across the longitudinal layers of the calorimeters in an area of $\Delta \eta \times \Delta \varphi=0.1 \times 0.1$
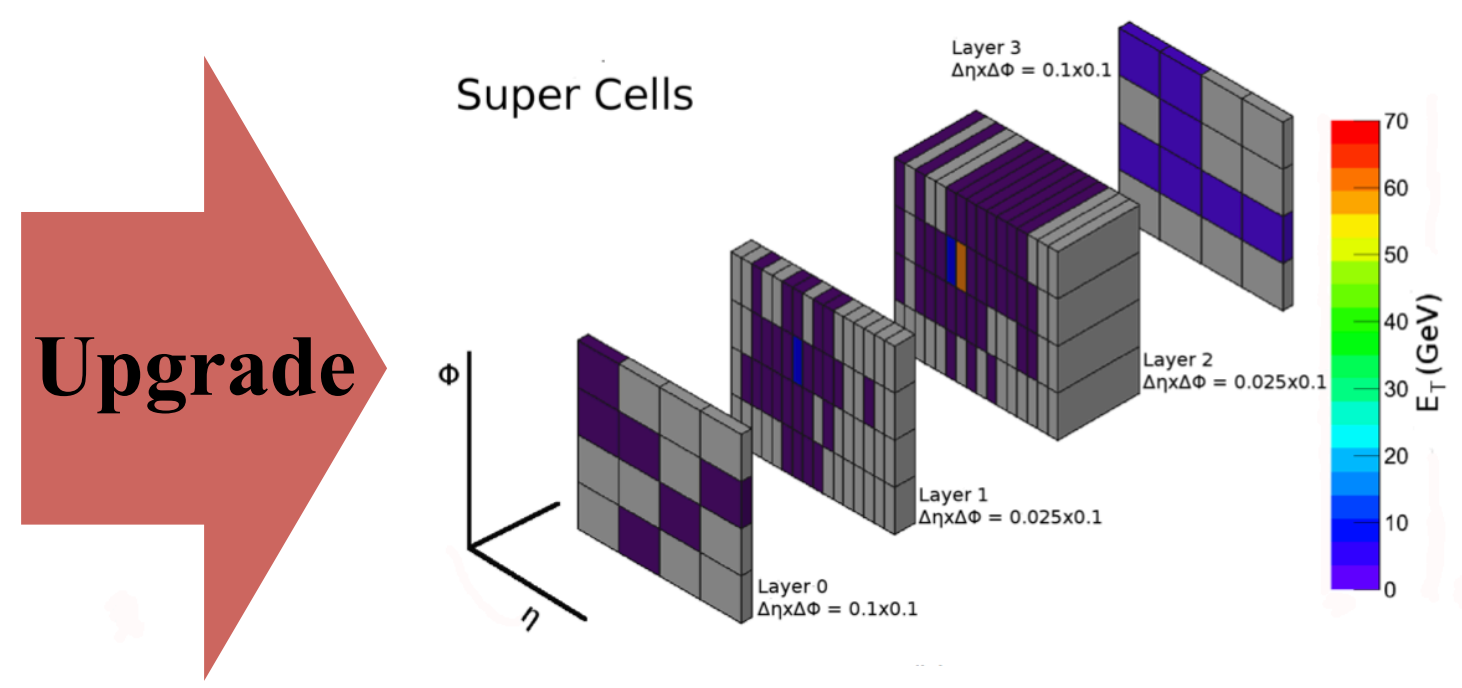

Super Cell :

provides information for each calorimeter layer for the full $\eta$ range of the calorimeter, and finer segmentation $(\Delta \eta \times \Delta \varphi=0.025 \times 0.1)$ in the front and middle layers 


\section{The Architecture of Upgraded Trigger Electronics}

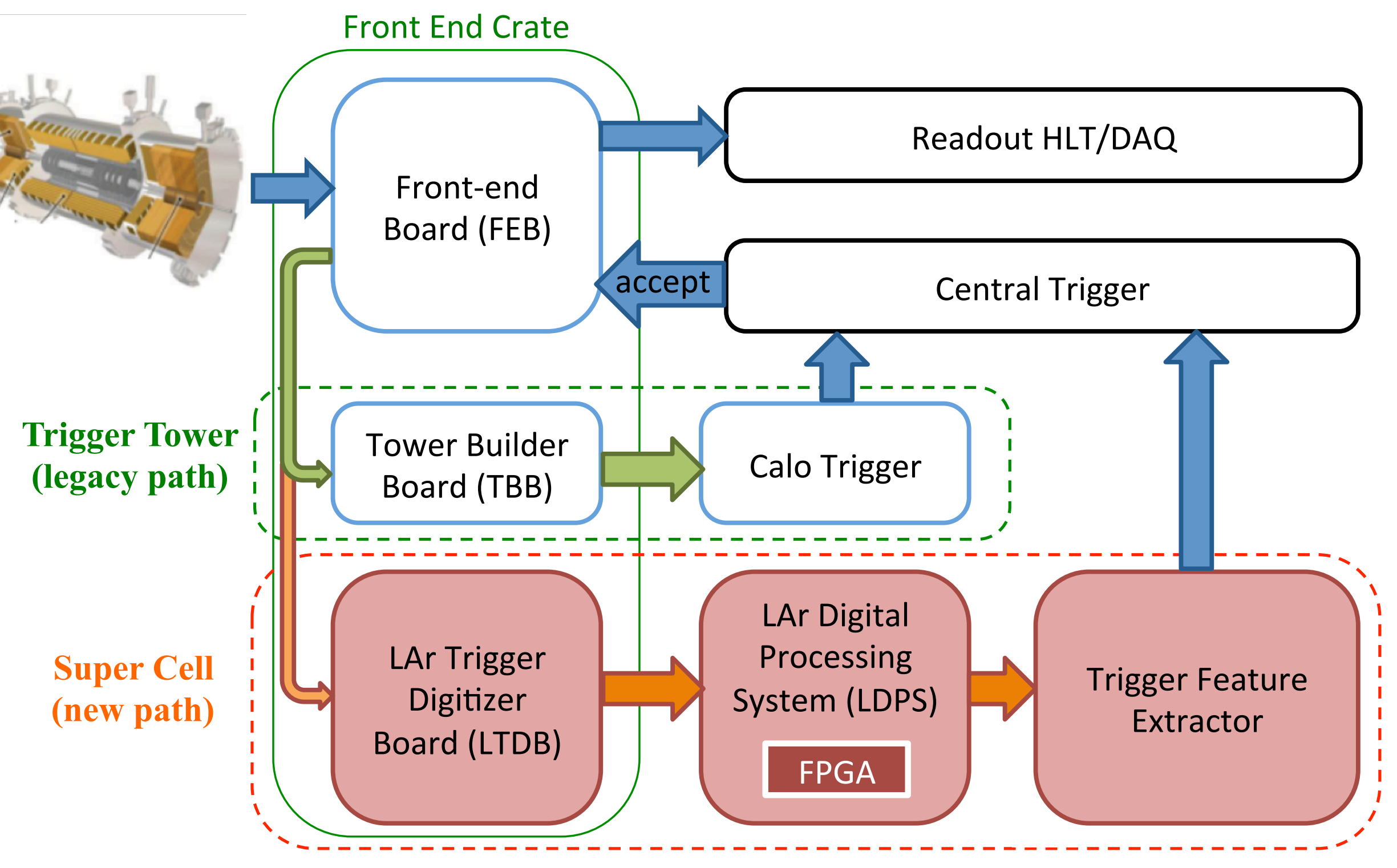




\section{LAr Trigger Digitizer Board (LTDB)}

To Tower Builder Board
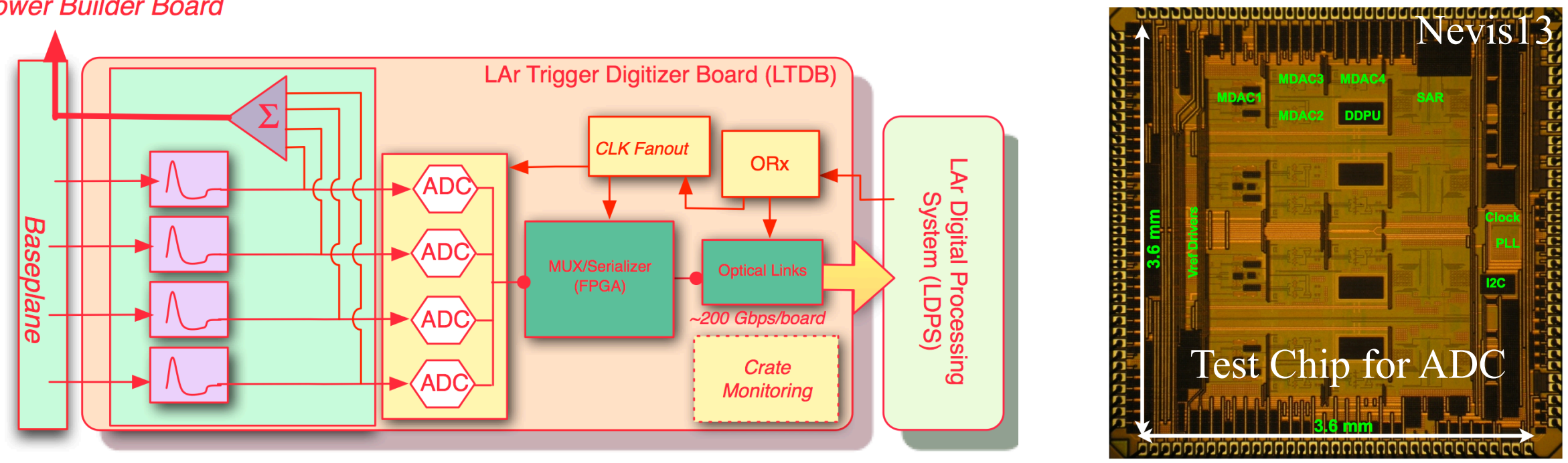

\section{The Role:}

- receive analog signal and digitize Super Cell signals

- Digitization is based on custom developed 12-bit SAR ADCs in $130 \mathrm{~nm}$ CMOS technology

- $40 \mathrm{MHz}$ sampling

- radiation tolerance

- transmit the digitized signal to the back end

- using the serializer (LOCx2) and optical driver (LOCld)

- $5.44 \mathrm{~Gb} / \mathrm{s}$ optical links per fiber (40 fibers in total)

- each of the 124 LTDBs handles up to 320 Super Cell channels

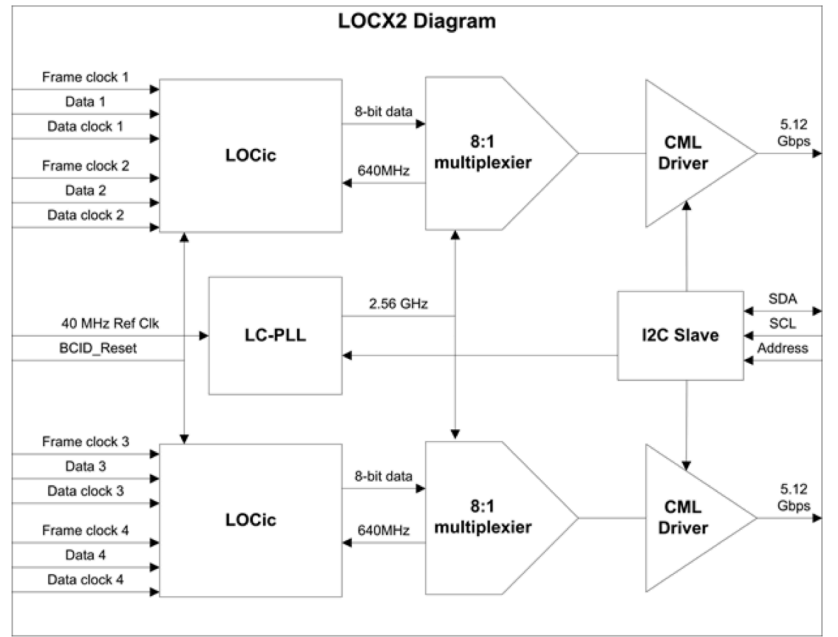




\section{LAr Digital Processing System (LDPS)}

\section{The Role:}

- receive the digitized data at a total rate of $25 \mathrm{~Tb} / \mathrm{s}$

- perform digital signal processing in real-time

- transmit the processed data to L1Calo at a total rate of $41 \mathrm{~TB} / \mathrm{s}$

\section{Design of LDPS:}

consist from 32 ATCA carrier blades

- which carry 4 mezzanine cards (AMC) each

- precise energy reconstruction, pile-up suppression and ID of the correct BC time are performed all on AMC

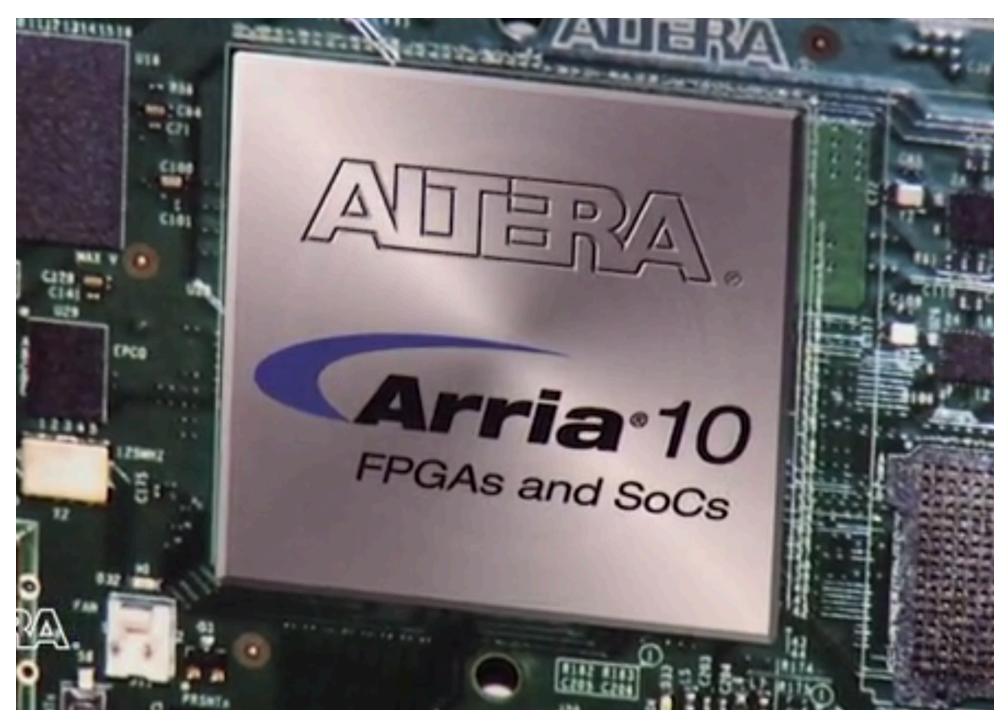

- real time processing done by ALTERA Arria-10

- each of which handles 1100 Super-Cells on average

Advanced Mezzanine Card (AMC)

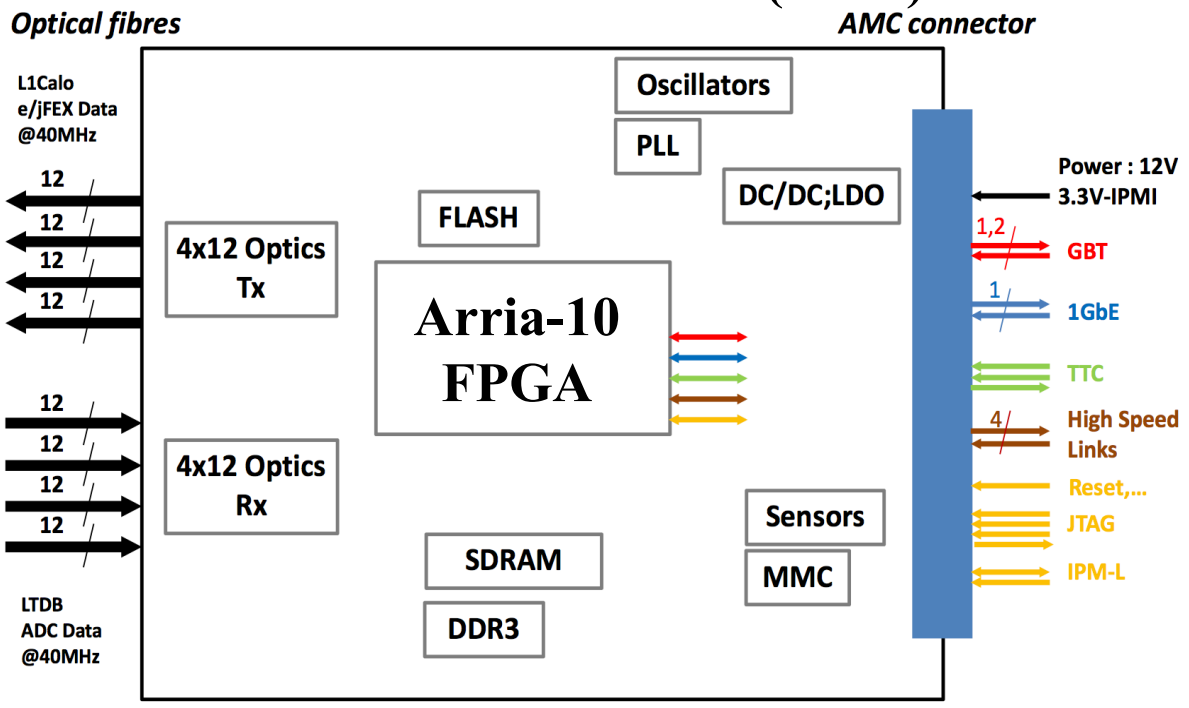

Carrier Board

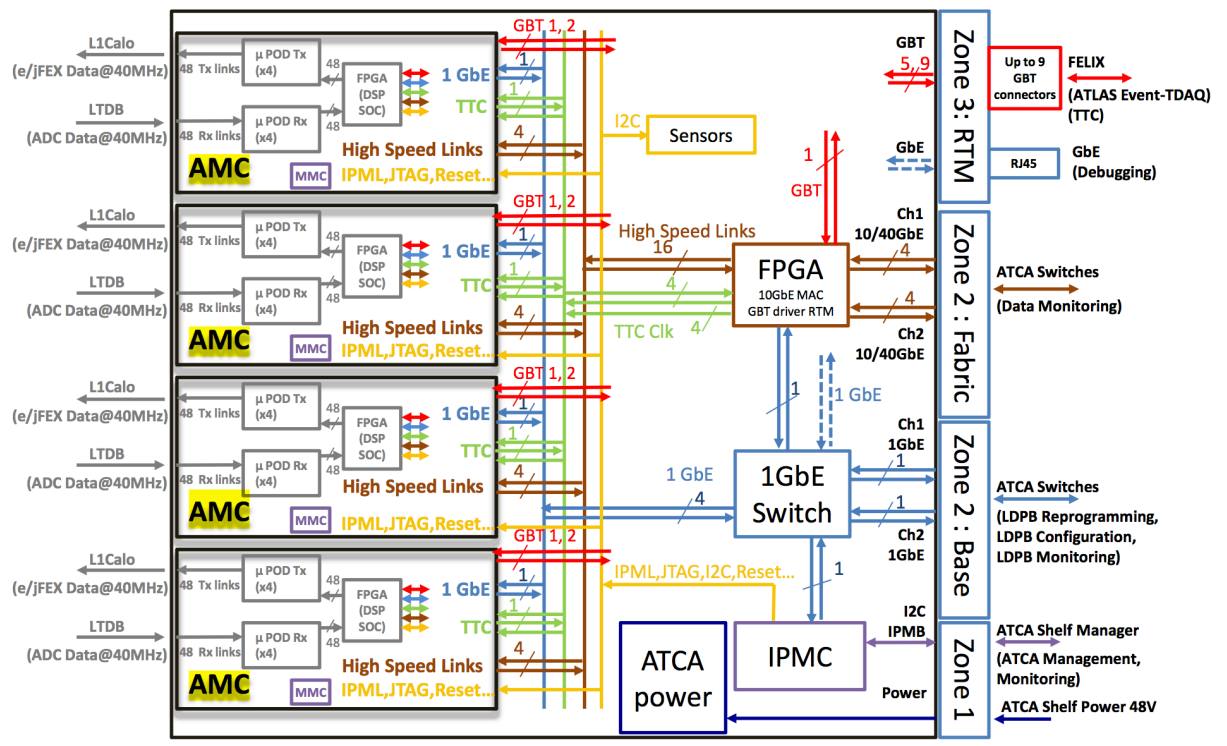




\section{Where Demonstrator is Installed}

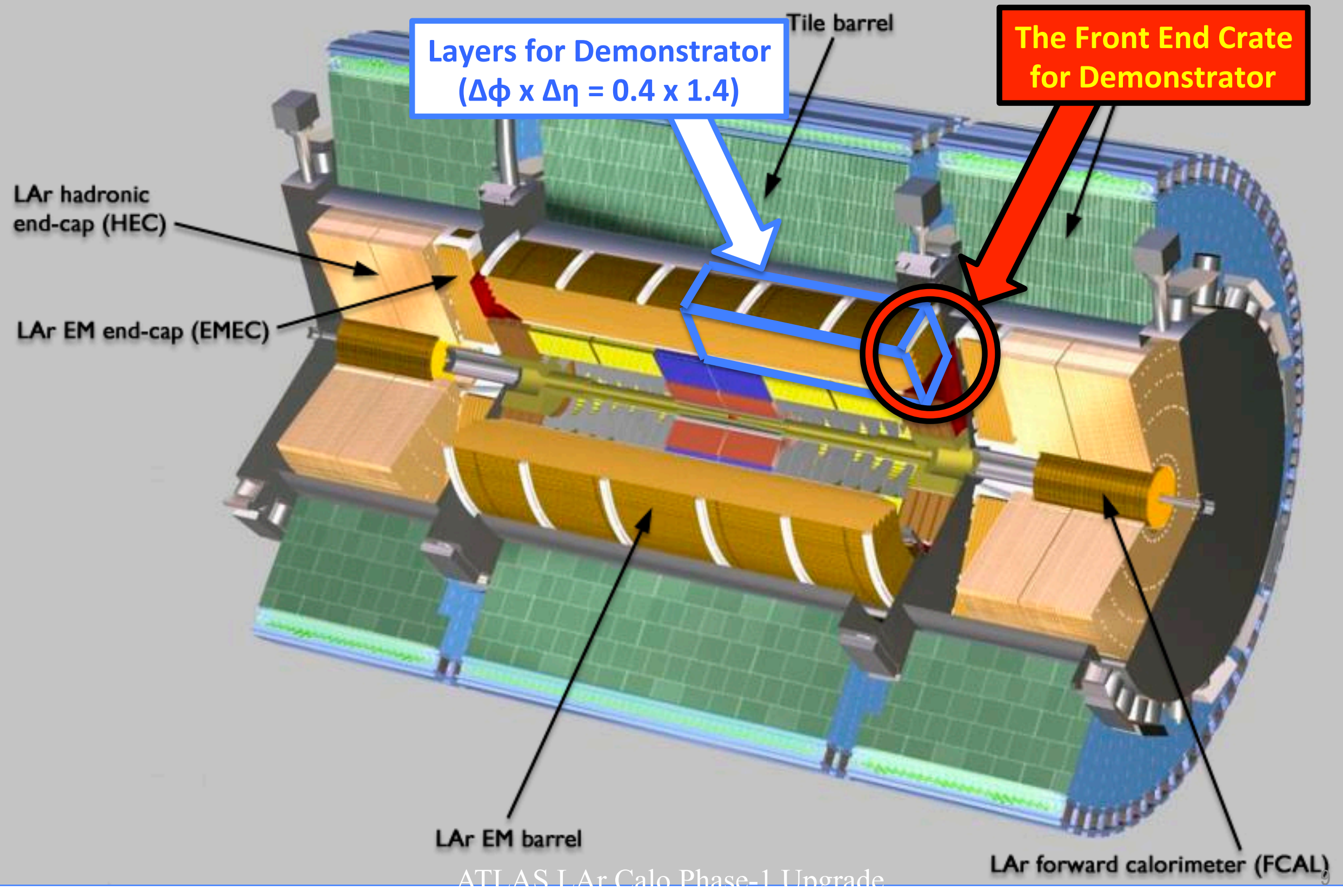




\section{Where Demonstrator is Installed}

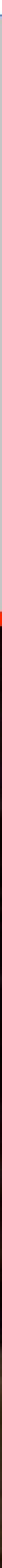




\section{Front View of Demonstrator Crate}

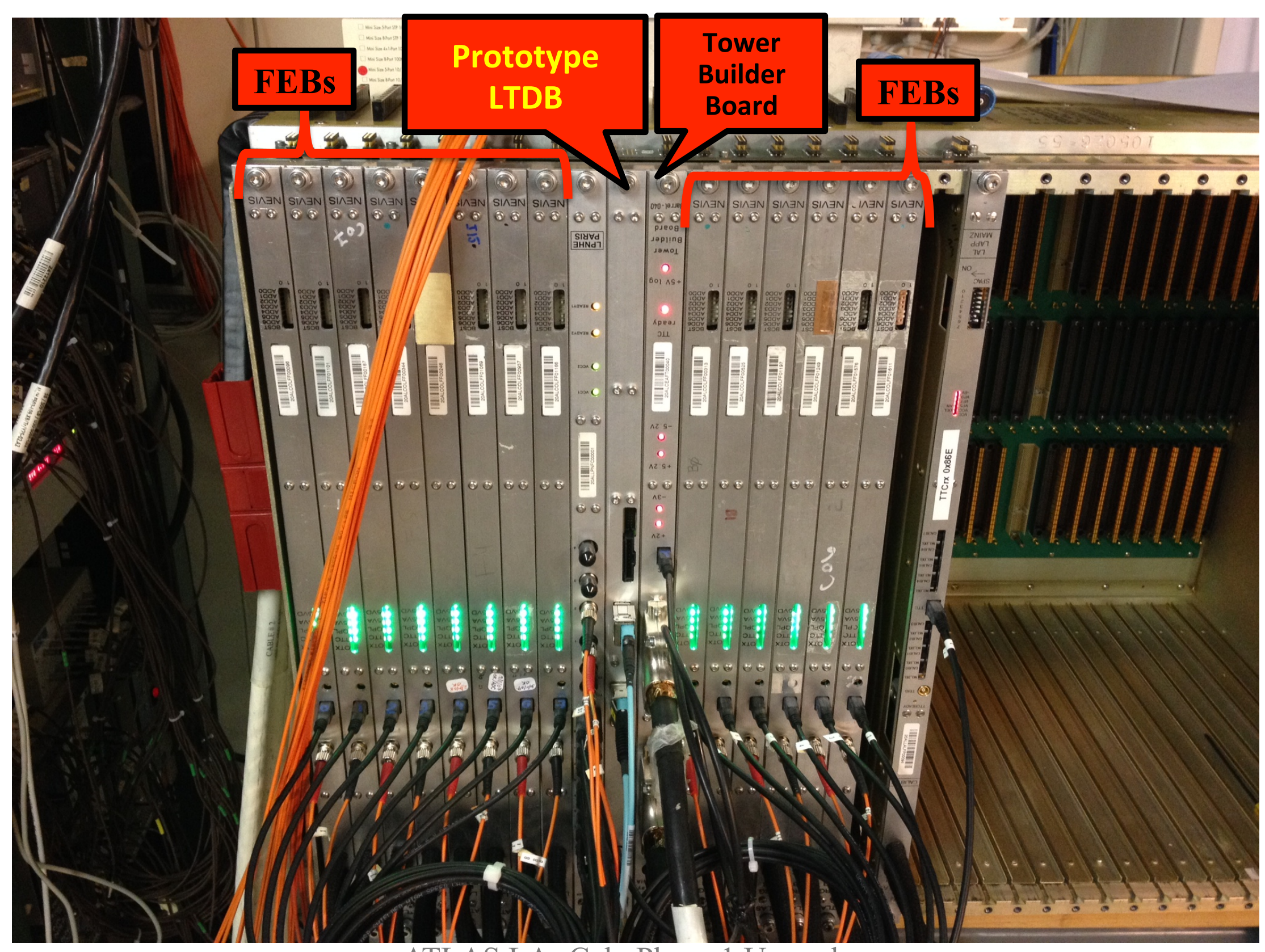




\section{The Prototype LDPB on Demonstrator}

- developed at LAPP

- The core components : ALTERA ${ }^{\circledR}$ Stratix IV FPGAs

- two Front FPGAs

- receive digitized data

The optical transceivers from the LTDB

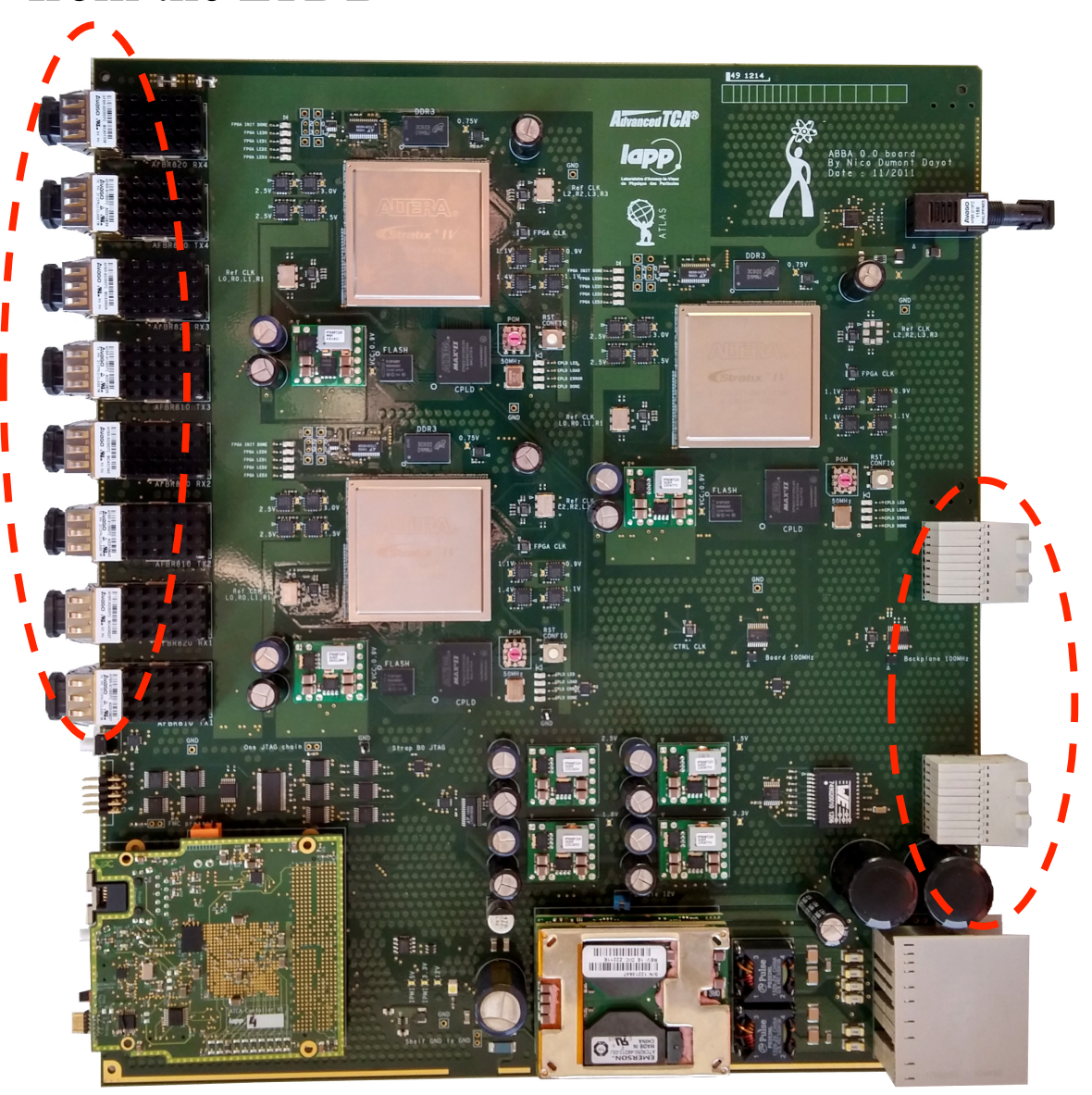

- format them in ATLAS RAW Event Format

- one Back FPGA

- readout through ATCA fabric interface with IPbus

- handle ICMP (Internet Control Message Protocol)

- interconnected via XAUI

The connectors to a $10 \mathrm{GbE}$ switch through ATCA backplane. The switch connected to PC with 2 optical fibers $(\mathrm{TX} / \mathrm{RX})$ at $10 \mathrm{GbE}$

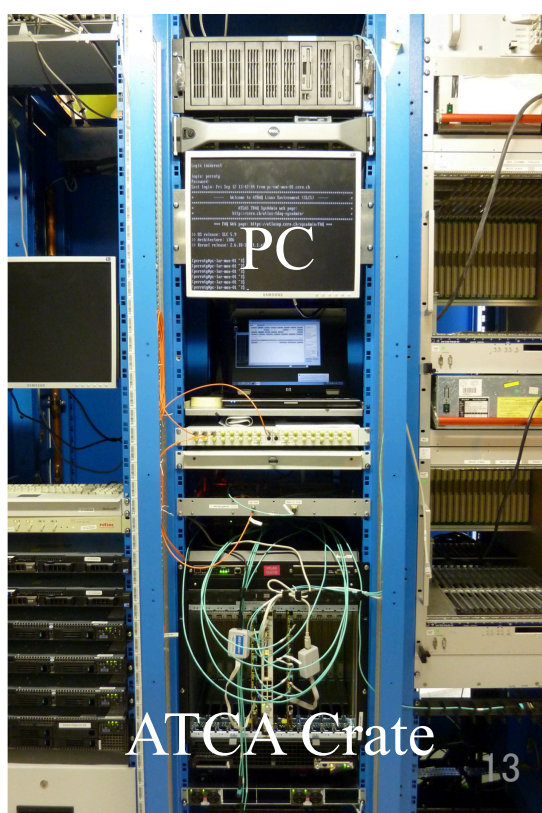




\section{Performance Results of}

Prototype Boards

in the Demonstrator System 


\section{Measurements on Demonstrator System}

On the legacy readout

- in order to verify no effect due to new electronics

- Total Noise \& Coherent Noise Fraction on Front End Boards

- Total Noise on the Trigger Readout for Run2

On the demonstrator readout

- Noise on LTDB Demonstrator

- Pulses from LTDB Demonstrator

- Linearity of LTDB Demonstrator 


\section{Total Noise on Front End Boards}

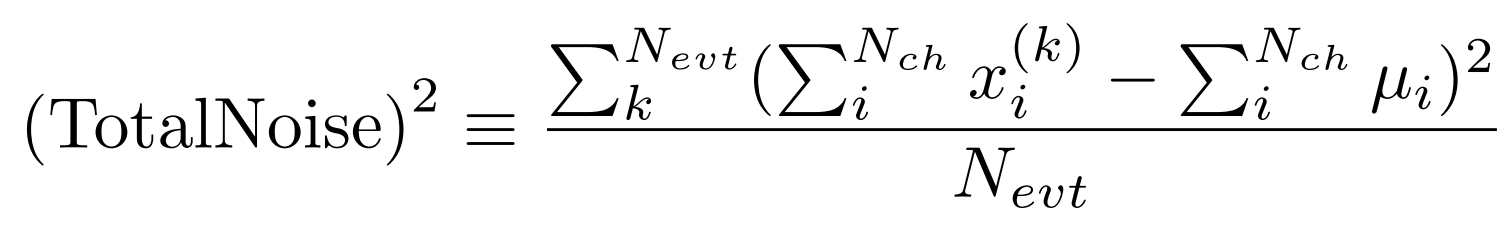

- The total noise of the 128 channels of the Front End Boards (FEBs)
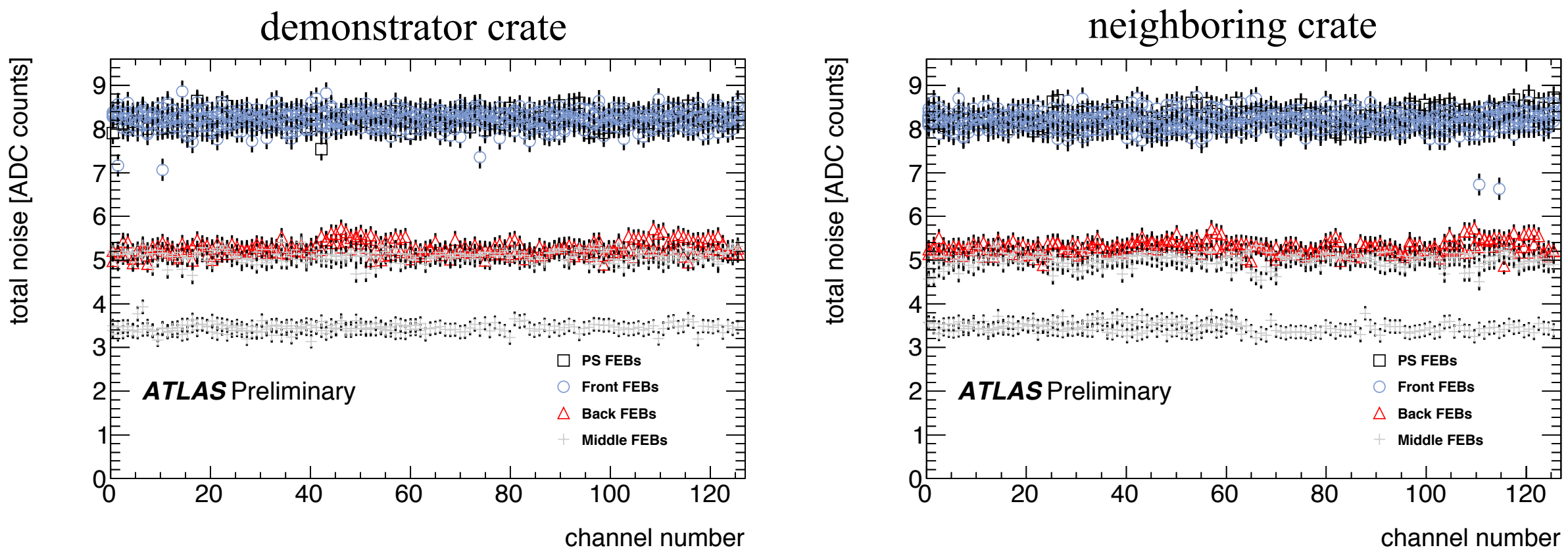

$\rightarrow$ The noise level of the demonstrator crate is consistent with that of neighboring crates 


\section{Coherent Noise Fraction}

\section{on Front End Boards}

- Coherent Noise Fraction $\left(\mathrm{CNF}: \rho_{\mathrm{COH}}\right)$ indicates how much noise of a channel originates from coherent noise in average

- The CNF for feedthroughs (FT) 7-12 on the detector has been computed

- FT9, $10 \rightarrow$ demonstrator crate

- FT7, 8, 11, $12 \rightarrow$ neighbor crates
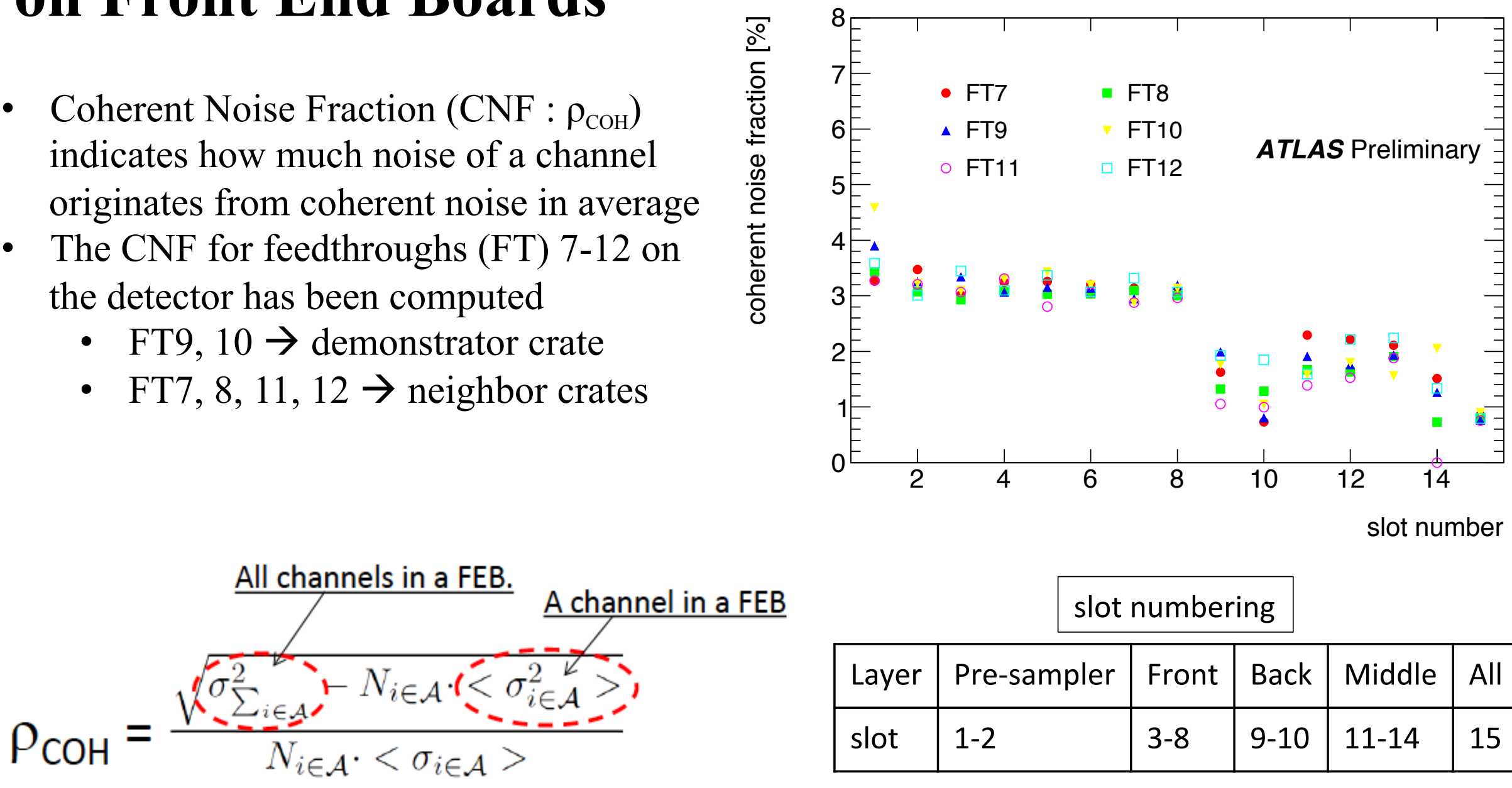

\begin{tabular}{|l|l|l|l|l|l|}
\hline \multicolumn{9}{|c|}{ slot numbering } \\
\hline Layer & Pre-sampler & Front & Back & Middle & All \\
\hline slot & $1-2$ & $3-8$ & $9-10$ & $11-14$ & 15 \\
\hline
\end{tabular}

$\rightarrow$ The noise level of the demonstrator crate is consistent with that of neighboring crates 


\section{Total Noise on the Trigger Readout for Run2}

- Total Noise on the Trigger Tower readout has been measured for confirming the demonstrator crate doesn't affect the trigger path used for physics@ Run2

$(\text { TotalNoise })^{2} \equiv \frac{\sum_{k}^{N_{e v t}}\left(\sum_{i}^{N_{c h}} x_{i}^{(k)}-\sum_{i}^{N_{c h}} \mu_{i}\right)^{2}}{N_{\text {evt }}}$

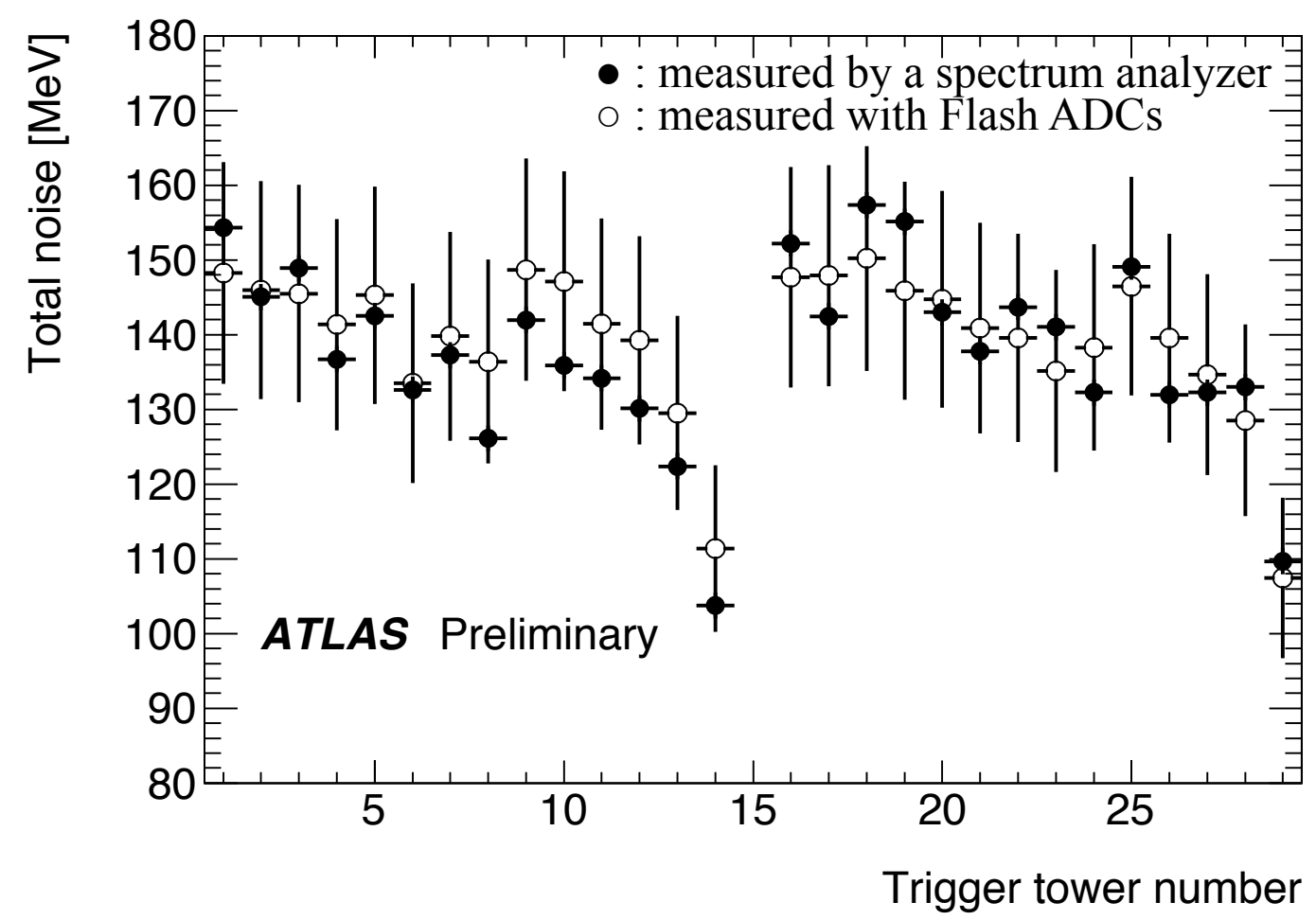

$\rightarrow$ Observed noise level is consistent with current system

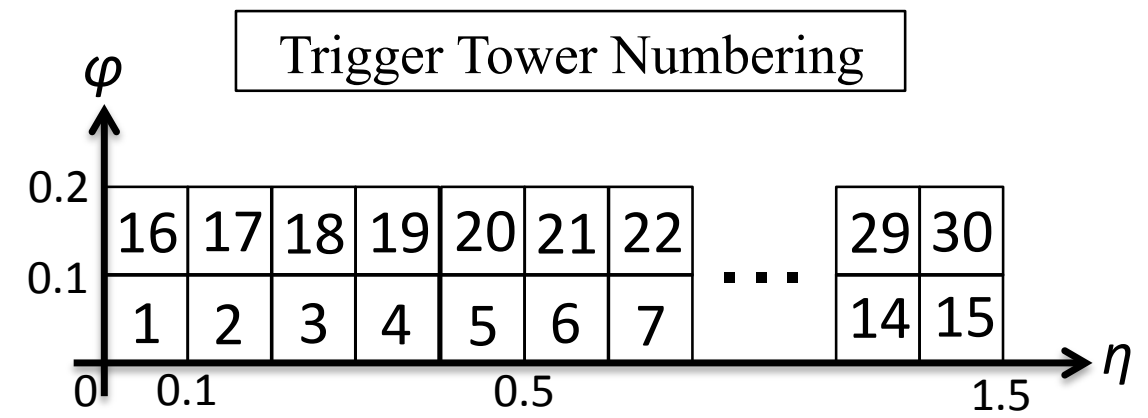




\section{On The Demonstrator Readout}




\section{Noise on LTDB Demonstrator}

- RMS of pedestal ADC of LTDB Demonstrator has been measured

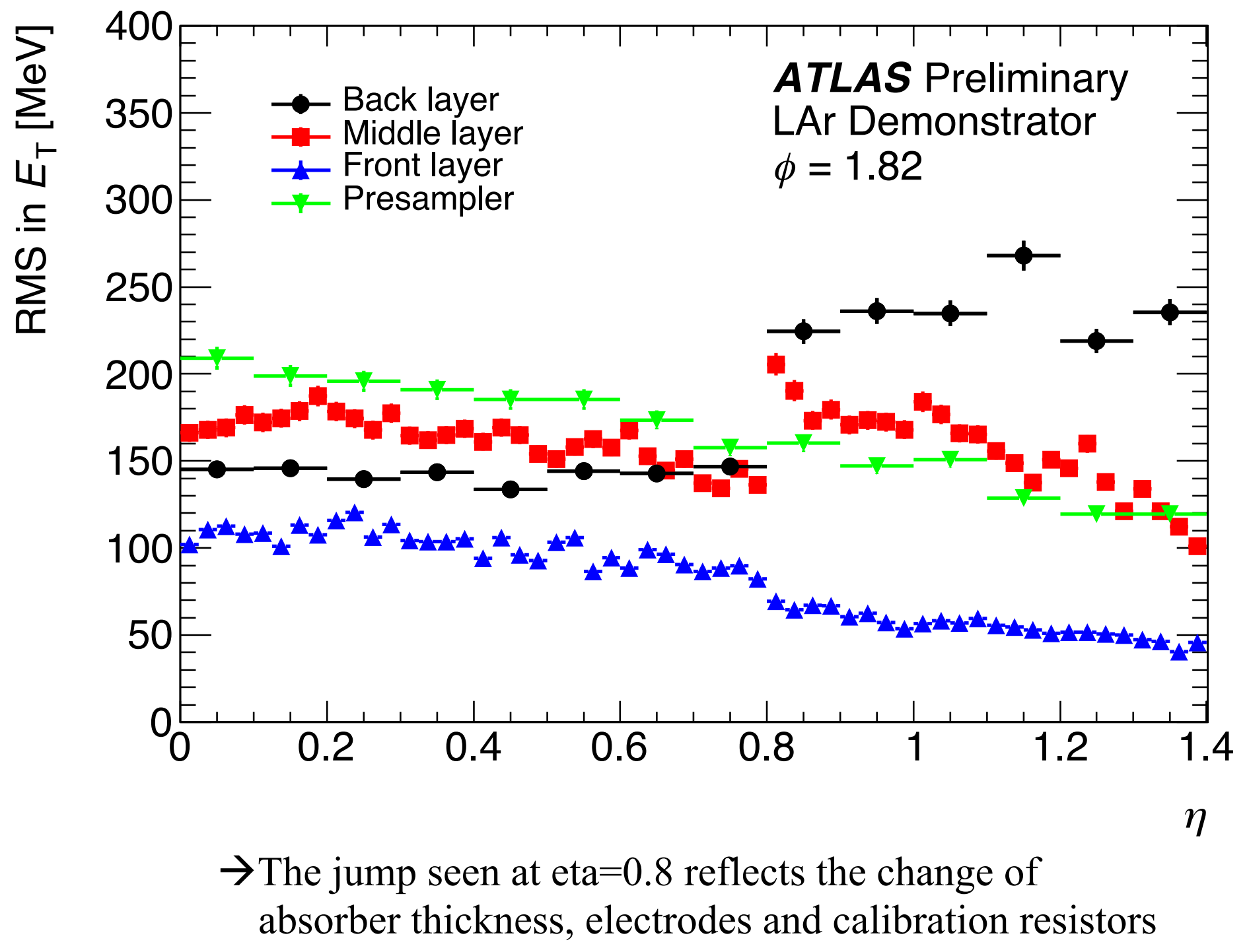




\section{Pulse from LTDB Demonstrator}

- Pulses have been seen from LTDB Demonstrator

- $\triangle \mathrm{ADC} \equiv \mathrm{ADC}_{\max }-\mathrm{ADC}_{\text {pedestal }}$

- $\mathrm{ADC}_{\text {pedestal }}$ is defined by the average of pedestal values from 0 to $50 \mathrm{~ns}$
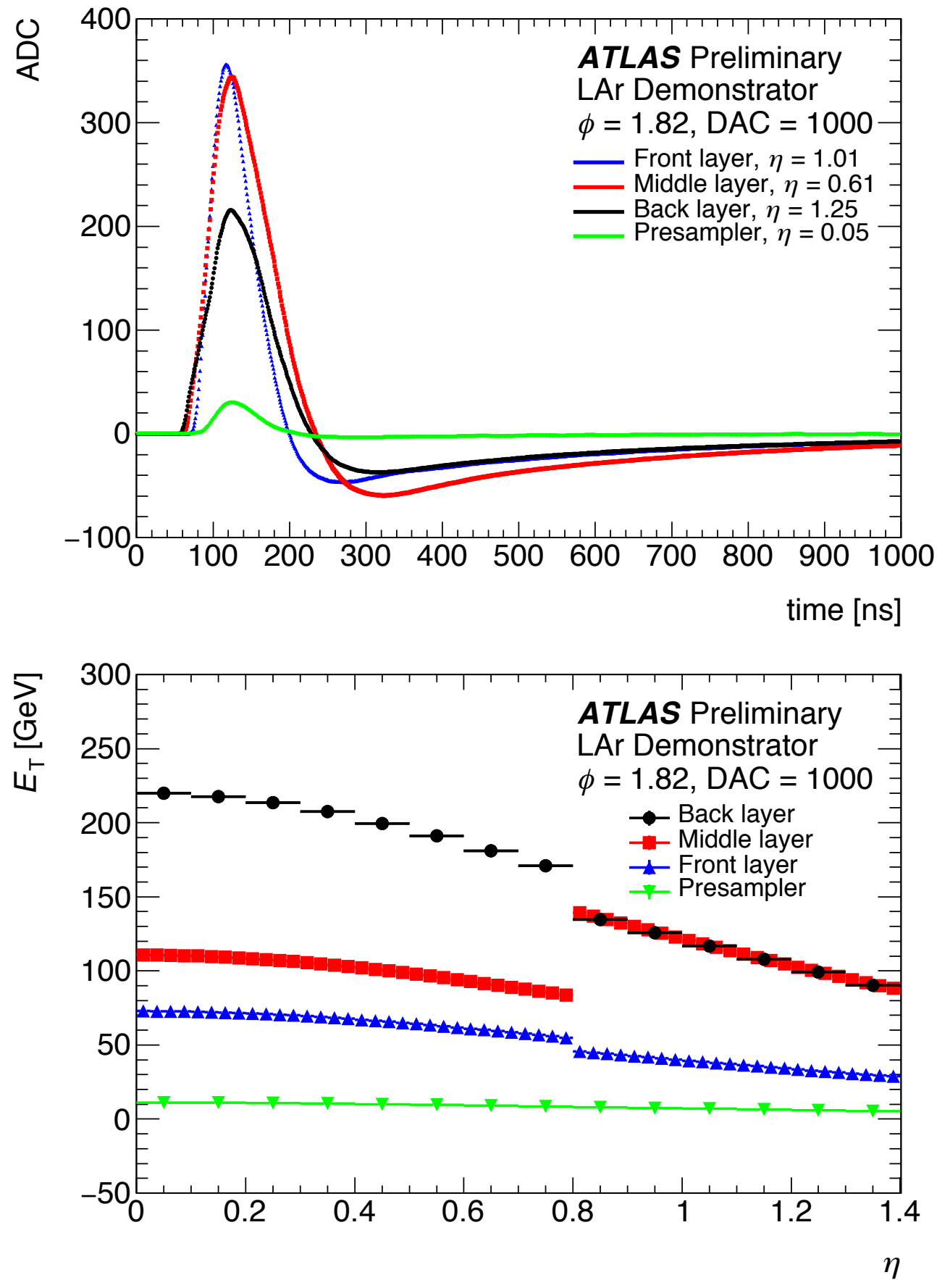


\section{Linearity of LTDB Demonstrator}

- Pulses have been checked with several DAC values

- Linearity has been checked as function of DAC

- Red down-triangle corresponds to the upper plot

- $\mathrm{ADC}$ is saturated @ $\mathrm{DAC}=10000$, but ADC count is not saturated $\rightarrow$ already saturated at analog readout $\rightarrow$ expected \& designed behavior
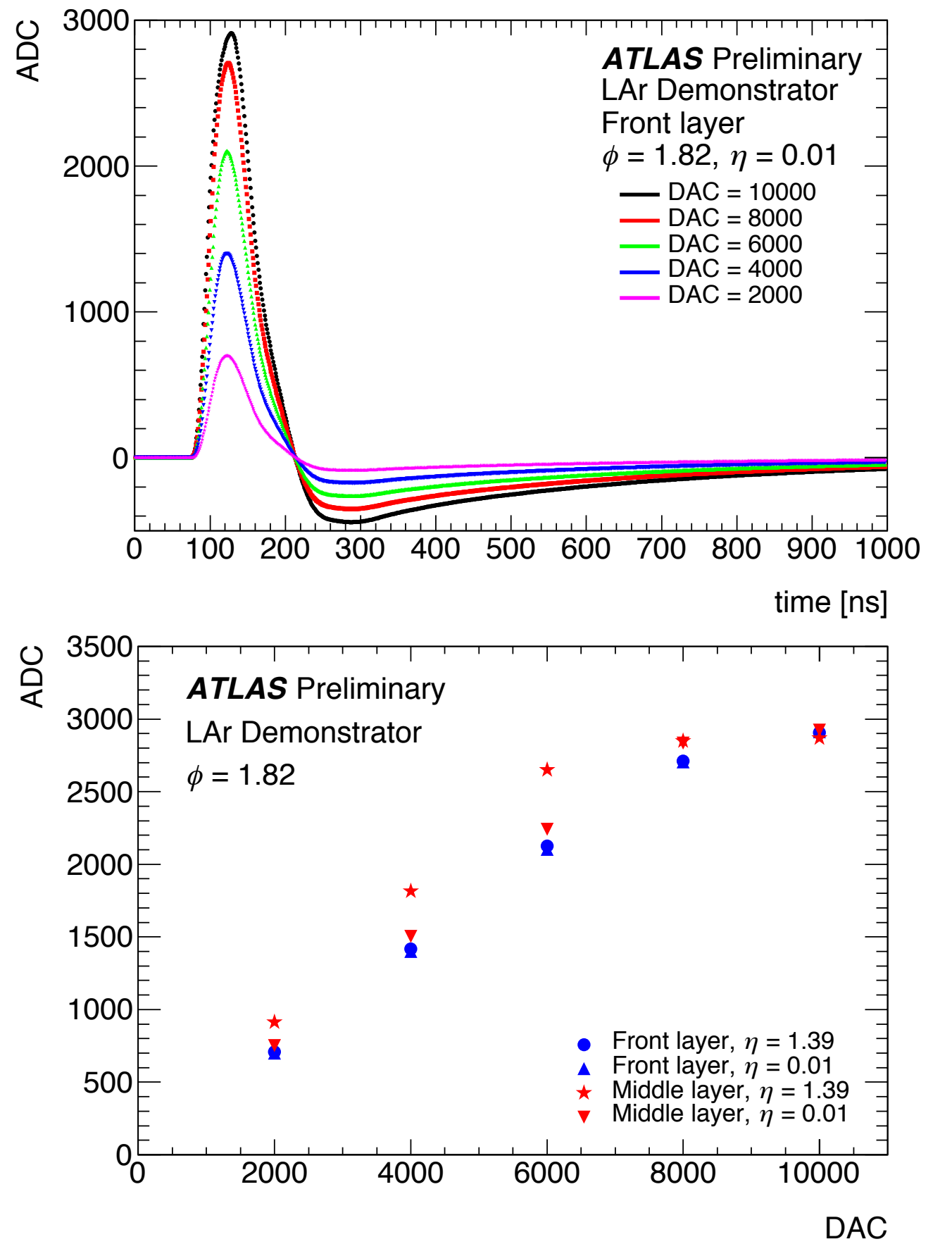


\section{Linearity of LTDB Demonstrator}

- Pulses have been checked with several DAC values

- Linearity has been checked as function of DAC

- Red down-triangle corresponds to the upper plot

- ADC is saturated @ DAC = 10000, but ADC count is not saturated $\rightarrow$ already saturated at analog readout $\rightarrow$ expected \& designed behavior $\rightarrow$ Linearity is kept up to high $\mathrm{E}_{\mathrm{T}}$ enough

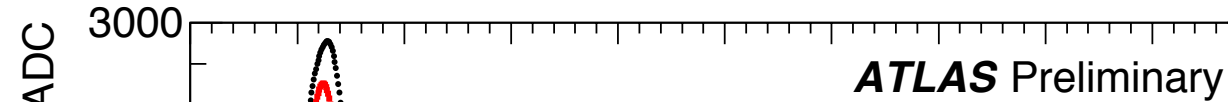

LAr Demonstrator Front layer

$\phi=1.82, \eta=0.01$

$-\mathrm{DAC}=10000$

- DAC $=8000$ DAC $=6000$

DAC $=4000$

DAC $=2000$

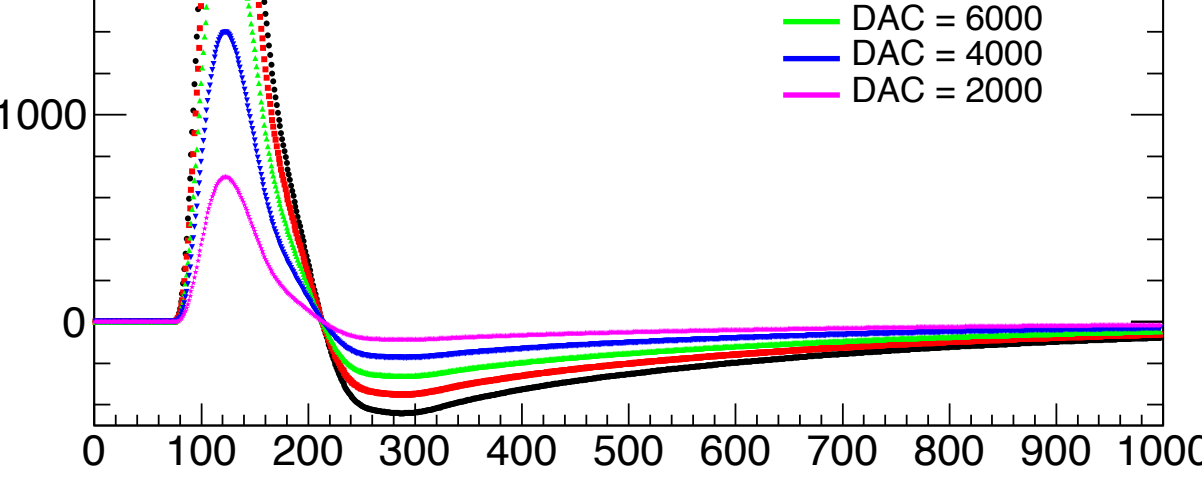

time [ns]

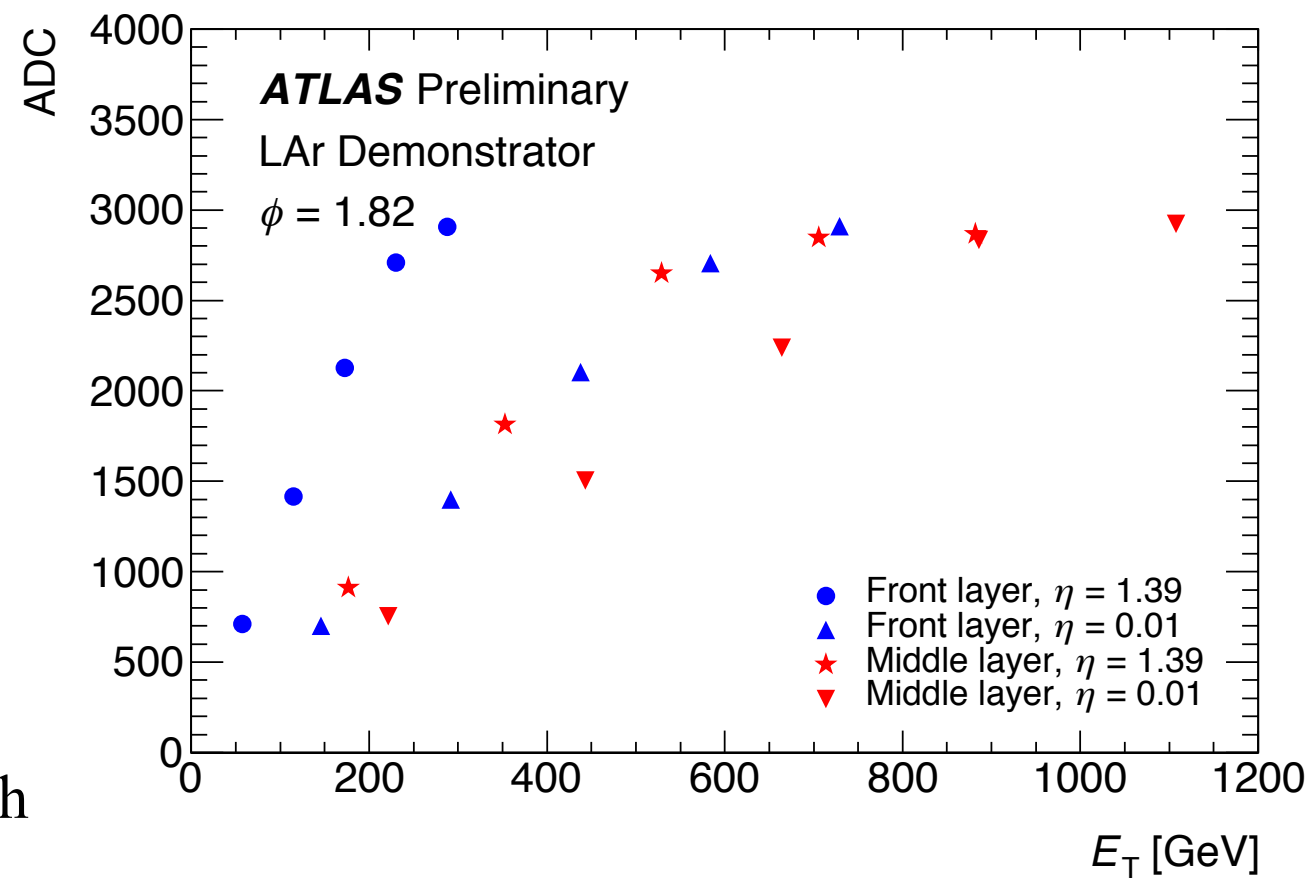




\section{$\underline{\text { Summary }}$}

- The performance of the prototype LTDB \& the prototype LDPB

on the demonstrator system has been measured;

- On the legacy readout

- confirmed to have no significant noise

- confirmed not to affect FEBs readout \& trigger path for Run2 significantly

- On the demonstrator readout

- pulse and noise have been observed

- linearity has been checked

\section{$\underline{\text { Plan }}$}

- Take the following data with pp collision for filtering algorithm for energy reconstruction

- Response of real EM object

- Noise data

$\rightarrow$ These are data for filtering algorithm development which we can not get without the demonstrator

Thank you very much! 
Backup 


\section{The Upgraded Trigger Electronics}

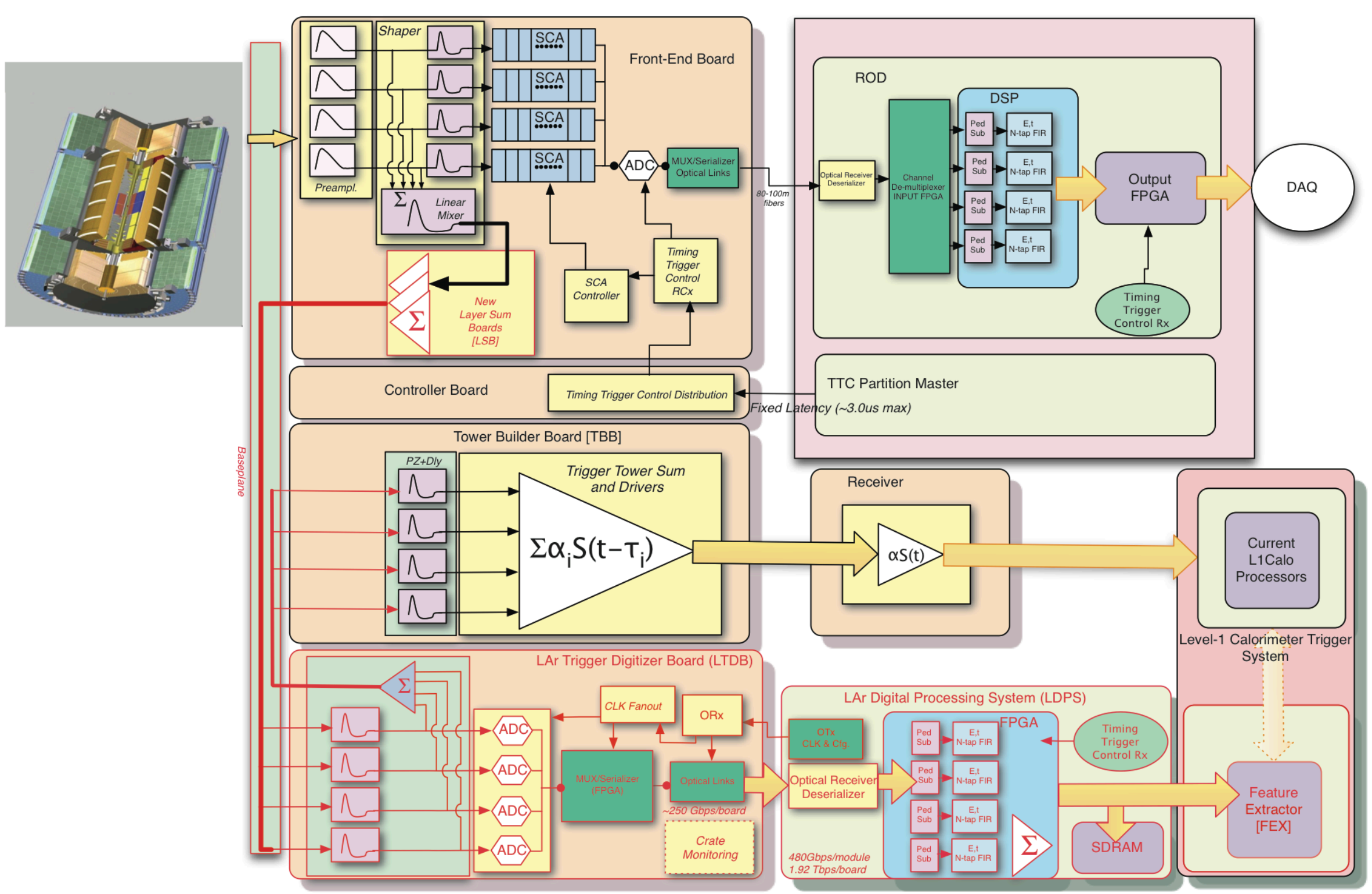

